\title{
Aeropuerto Río Amazonas y su realidad operativa en el turismo de Pastaza
}

Río Amazonas Airport and its operational reality in the Pastaza tourism

Bryan Steven González Piedra. ${ }^{1}$, Edison Eduardo Ruiz Mármol. ${ }^{2}$, Mayra Inés Pinta Rodríguez. ${ }^{3} \&$ Clímaco Geovani Espín Ortiz. ${ }^{4}$

\begin{abstract}
.
DOI: $\underline{\text { https://doi.org/10.33262/concienciadigital.v4i2.2.1783 }}$

Mobility is essential for all economic and social activities. For this reason, from the fifties, air traffic began its massive generalization, becoming a means of transport for all groups in society. In Ecuador, according to the Andean Community, in 2019, passenger air traffic has increased, however, in the Amazon region, the limited information regarding the benefits and services offered by the Amazonian airports blur the real performance that they have. and its operational reality. This research analyzes the reality of the Rio Amazonas Airport as a potential means of passenger mobilization, and its potential to join the tourism system of the Ecuadorian Amazon. The data shown here is based on the information provided by the General Directorate of Civil Aviation located in the Shell parish, Pastaza province where the files are located. The type of research is descriptive of a cross-sectional quantitative nature for the collection of secondary information and the application of the structured questionnaire to determine its perception. Its infrastructure, which is operational and underused, is highlighted, in addition to the fact that the services of the Río Amazonas Airport serve in many cases of social assistance, so it is a prop for

\footnotetext{
1 Universidad Estatal Amazónica, Maestrante Centro de Postgrados, Pastaza, Ecuador. bs.gonzalezp@uea.edu.ec, https://orcid.org/0000-0003-4715-0674

2 Universidad Estatal Amazónica, Facultad Ciencias de la Vida, Pastaza, Ecuador. eruiz@uea.edu.ec, https://orcid.org/0000-0001-8968-8814

3 Universidad Estatal Amazónica, Centro de Postgrados, Pastaza, Ecuador. mpinta@uea.edu.ec, https://orcid.org/0000-0002-8285-5381

${ }^{4}$ Universidad Estatal Amazónica, Facultad Ciencias de la Vida, Pastaza, Ecuador. cespin@uea.edu.ec, https://orcid.org/0000-0001-5047-4704
} 
the development of the communities in the interior of the Amazon jungle that are due to activities of visitation, it is important to mention that the airport is mostly used for domestic activities and others, scarcely for tourism. The Rio Amazonas Airport has all the facilities to operate with a greater influx of passengers, however, it is not considered in the offer.

Keywords: Air passenger traffic, tourist demand, tourism in the Amazon, Shell Mera

\section{Resumen}

La movilidad es indispensable para todas las actividades económicas y sociales. Por ello a partir de los años cincuenta el tráfico aéreo comenzó su generalización masiva, convirtiéndose en un medio de transporte para todos los colectivos de la sociedad. En Ecuador según la Comunidad Andina (2019) el tráfico aéreo de pasajeros se ha incrementado, sin embargo, en la región Amazónica, la limitada información respecto a las bondades y servicios que ofrecen los aeropuertos de la amazonia desdibujan el real desempeño que ellos tienen y su realidad operativa. La presente investigación analiza la realidad del Aeropuerto Rio Amazonas como potencial medio de movilización de pasajeros, y su potencialidad para incorporarse al sistema turístico de la amazonia ecuatoriana. Los datos aquí mostrados se amparan en la información proporcionada por la Dirección General de Aviación Civil ubicado en la parroquia Shell provincia de Pastaza donde reposan los archivos. El tipo de investigación es descriptiva de carácter cuantitativo transversal para la recolección de información secundaria y la aplicación del cuestionario estructurado para determinar su percepción. Se desatacan su infraestructura que se encuentra operativa y subutilizada, además que los servicios del Aeropuerto Río Amazonas sirven en muchos casos de ayuda social, por lo que es un puntal para el desarrollo de las comunidades del interior de la selva amazónica que se deben a actividades de visitación, es importante mencionar que en su mayoría el aeropuerto es utilizado por motivo de actividades domésticas y otros, escasamente por turismo. El Aeropuerto Río Amazonas dispone de todas las facilidades para operar con mayor afluencia de pasajeros, sin embargo, no es considerado en la oferta.

Palabras claves: Tráfico aéreo de pasajeros, demanda turística, turismo en la amazonia, Shell Mera

\section{Introducción}

La movilidad es indispensable para todas las actividades; a partir de los años cincuenta el tráfico aéreo comenzó su generalización masiva, convirtiéndose en un medio de transporte seguro, de rápido desplazamiento y bajos costos (Rodrigue, Comtois, \& Slack, 2013).

La Organización Mundial del Turismo (2012) en la vigésima reunión de la Asamblea General señala que la aviación ha tenido un fuerte efecto multiplicador en la economía debido a la llegada de los pasajeros internaciones ya sea por turismo, negocios y migración; que generan puestos de empleo y exportaciones. 
En el Ecuador ha incrementado el tráfico aéreo de pasajeros según la Comunidad Andina (2019), en la región Amazónica desde el Aeropuerto Río Amazonas ingresan personas y carga hasta las comunidades asentadas en la selva, sin embargo, la limitada información existente dificulta el desarrollo de las actividades comerciales, especialmente las de turismo.

Este aeropuerto cuya pista fue construida en los años 40, ubicado en la parroquia Shell, sirve a los pobladores de la provincia de Pastaza y otras, que tienen déficit de vías de comunicación terrestre y fluvial; a través de este se ingresan productos básicos, brigadas de salud, medicina, colación escolar, vuelos del Aero policial y transporte logístico de las Fuerzas Armadas, o salen a los centros urbanos más cercanos a comercializar productos agrícolas, maderas, pesca y artesanías afanosamente trabajadas en las comunidades (Dirección General de Aviación Civil, 2020).

Por la importancia económica y social de este aeropuerto varias compañías comerciales de transporte no regular como AEROSARAYAKU, AEROKASHURKO, SAMAFE, AEROFOR y AEROCONEXOS, Grupo Aéreo del Ejército, la Fuerza Aérea y la Base del AEROPOLICIAL lo utilizan, incluido TAME Amazonia en su tiempo de operaciones; además en sus instalaciones se desarrollan las actividades de la Escuela de Aviación Pastaza y la Escuela AEROSERTEC donde se forman pilotos comerciales (Aerosertec, 2020).

La presente investigación, amparada en la información entregada por la Dirección General de Aviación Civil, presenta la caracterización general del Aeropuerto Río Amazonas, misma que permite establecer su magnitud y características específicas de operación. Posterior a ello, se procede con la fase empírica con un cuestionario estructurado en relación al motivo del uso del aeropuerto condensado por medio del catalizador turismo motivo del presente estudio. Conocer la realidad operativa del Aeropuerto Río Amazonas permite generar información estadística suficiente, formal y constante con la intención de orientar a los actores de la actividad turística en sus decisiones (Boullón, 2006).

\section{Metodología Características generales del lugar}

La investigación se realizó en la Provincia de Pastaza en el cantón Mera, creado el 10 de abril de 1967, en la administración del presidente Otto Arosemena Gómez, este cantón tiene una superficie de $601.1 \mathrm{~km}^{2}$, la población es de 11.861 habitantes (INEC, 2020), en su organización territorial tiene 3 parroquias Mera, Shell y Madre Tierra, cuenta con una altitud de $1.150 \mathrm{msnm}$. Este cantón limita al norte con la provincia de Napo, al sur por la provincia de Morona Santiago, al este los cantones de Pastaza y Santa Clara, al oeste con las provincias de Morona Santiago y Tungurahua, su clima es cálido húmedo, con una temperatura de $20.2^{\circ} \mathrm{C}$, su precipitación es $4222.7 \mathrm{~mm}$. (promedio anual) y con una densidad de 19.7 hab./km2. El 91,7 \% reside en el área rural; se caracteriza por ser una población joven con el 47,5\% de menores de 20 años. 


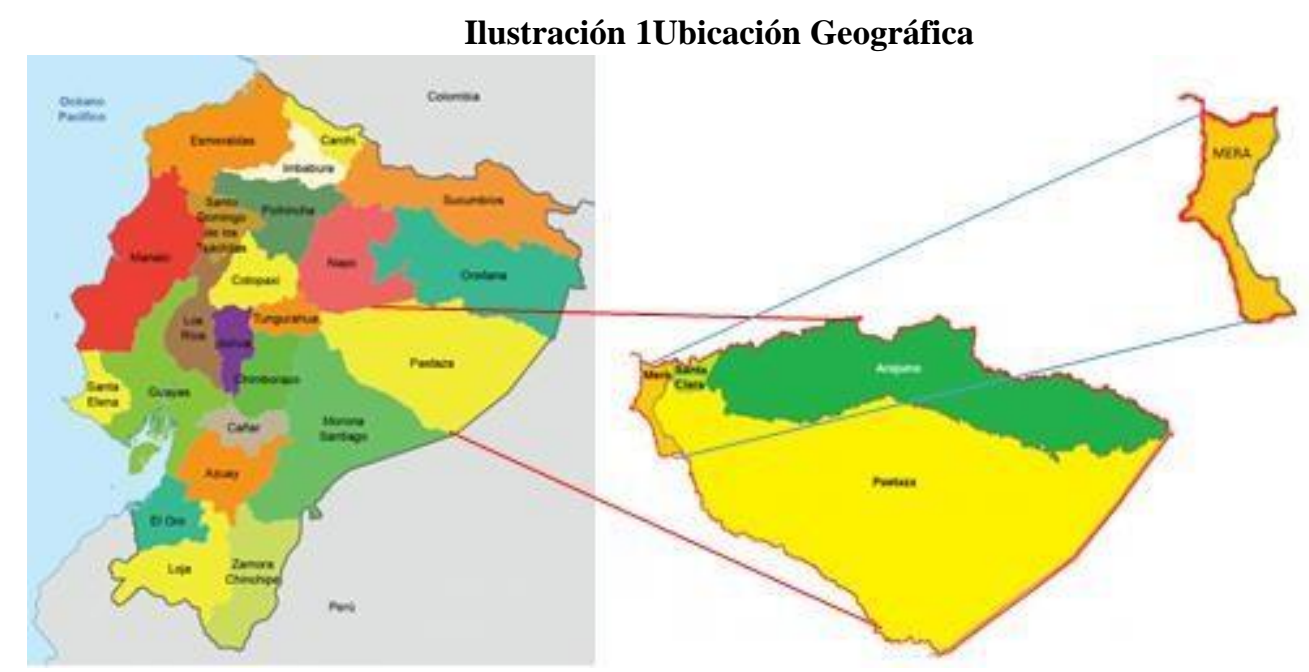

Fuente: Ubicación geográfica del sitio de estudio.

El Aeropuerto Río Amazonas SESM según el Código de Aeropuertos de OACI Organización de Aviación Civil Internacional (Dirección General de Aviación Civil, 2020), se encuentra ubicado en la parroquia Shell, en las siguientes coordenadas $01^{\circ} 30^{\prime} 19$ 'S, $78^{\circ} 03^{\prime} 45^{\prime}$ W W la misma que limita con el Fuerte Militar Amazonas, Brigada de Selva N.- 17 BS-Pastaza, labora los 365 días del año.

La ciencia puede ser entendida como un sistema de conocimientos demostrados, que proceden de acuerdo con un método y que se utiliza por el ser humano pasa describir y explicar los fenómenos que observa, de acuerdo a leyes y principios científicos (Eyssautier, 2006). La técnica es un conjunto de instrumentos de medición elaborados en base a los conocimientos científicos; el instrumento no es necesariamente un aparato mecánico o electrónico, puede ser un inventario, un test, una entrevista, un cuestionario, un formulario, un cálculo estadístico, un trabajo en grupos; en fin es una herramienta que se usa en la investigación (Barrantes, 2016). Del mismo modo se utilizó el método deductivo, a manera de estrategia de reflexión, siendo adecuado para analizar objetivamente los datos recopilados y obtener conclusiones lógicas (Fernández, Baptista, \& Hernández, 2006). En este contexto los autores indican que "los estudios se realizan de un hecho, fenómeno, individuo o grupo con el fin de establecer su estructura o comportamiento".

La presente investigación se muestra bajo dos enfoques, en diferentes momentos así, enfoque de carácter cualitativo en la caracterización general del Aeropuerto Río Amazonas y el enfoque cuantitativo en la valoración de la encuesta que llevará a establecer la realidad del tráfico aéreo de pasajeros con respecto al turismo de la provincia y la amazonia en general.

La investigación tuvo un diseño estadístico, de tipo descriptivo y transversal, donde el investigador se enfoca en medir la presencia, características y distribución de un fenómeno en una población en un momento determinado, sin la intención de establecer relaciones causales con otros factores referido a algo en concreto (Veiga, De la Fuente, \& Zimmermann, 2008), como en este caso la movilidad por motivos de turismo y uso del aeropuerto para su desplazamiento, esta medición permitió estructurar la información de 
las variables de acuerdo a la percepción de la demanda en primera línea, que alimentó la data de manera valiosa acorde a la realidad de uso del aeropuerto. Al ser transversal recoge los datos para detallar la frecuencia (Mata 2019) de uso del aeropuerto por motivos de turismo, siendo que el desplazamiento no es directamente relacionado con actividades de ocio. El enfoque investigativo fue cuantitativo y cualitativo. El presente trabajo se encuentra en el Nivel Descriptivo, presentando un tipo de estudio con diseño estadístico, aplicando un cuestionario estructurado por medio de la plataforma office 365 Forms, aplicado a los usuarios del servicio de transporte aéreo del Aeropuerto Río Amazonas en la cantidad de 385 usuarios, con un $95 \%$ de confiabilidad y $5 \%$ de error con un universo desconocido.

\section{Resultados}

\section{Caracterización del Aeropuerto Río Amazonas}

Según datos de la Dirección de Aviación Civil, DAC (2020), el medio de acceso y salida más utilizado en las comunidades del interior de la amazonia ecuatoriana es el aéreo, desde el Aeropuerto Río Amazonas ingresan personas y carga a los asentamientos de las siete nacionalidades de la selva amazónica: Achuar, Andoa, Shuar, Kichwa, Shiwiar, Waodani, Zápara, aproximadamente a trescientas veinte comunidades que habitan en estos sectores.

Este aeropuerto, sirve a la población de la provincia de Pastaza y otras amazónicas que tienen déficit en lo relacionado con vías de comunicación; apoyando de esta manera a los núcleos humanos que habitan en estas zonas quienes no han perdido su identidad, costumbres, lengua y cultura, y que desarrollan su vida diaria en el pulmón de la humanidad, conocido también como un laboratorio biológico del mundo (Visitaecuador, 2020).

La pista existente de pavimento flexible fue construida en el año 1940 por la Compañía Petrolera Shell con la finalidad de apoyar al desarrollo de las actividades en la zona, posteriormente, la Dirección General de Aviación Civil con el paso de los años fue implementando poco a poco los diferentes servicios aeronáuticos con los que actualmente dispone, lo que ha beneficiado a la seguridad operacional.

La pista dispone de todos los servicios aeronáuticos y radioayudas para la navegación, permitiendo que las operaciones aéreas se realicen con altos índices de seguridad, cumpliendo con las leyes y regulaciones existentes, logrando armonizar el control que ejerce la institución sobre el transporte aéreo.

Su superficie total es de 31,28 hectáreas, con una pista de $1487 \mathrm{~m}$. de longitud, ancho de $23 \mathrm{~m}$, superficie con asfalto flexible; y una plataforma de $17250.00 \mathrm{~m}^{2}$, con $230 \mathrm{~m}$. de longitud y $75 \mathrm{~m}$. de ancho.

Es un aeropuerto de tipo civil, administrado por la Dirección General de Aviación Civil y cuya dirección está a cargo del Administrador Aeroportuario de Shell quien cuenta con la colaboración de 55 servidores públicos que laboran los 365 días del año en horario HJ, 
es decir, desde la salida del sol hasta su puesta, mediante NOTAM C0599/20 desde las 08:00 hasta las 18:00 horas brindando los servicios de:

1. Tránsito aéreo,

2. Operaciones aeronáuticas,

3. Información aeronáutica (AIS),

4. Búsqueda y salvamento (Subcentro SAR-RSC Amazonas),

5. Extinción de incendios,

6. Seguridad aeroportuaria,

7. CNS,

8. Vigilancia continua regional III y

9. Abastecimiento de combustible

En el año 2014, la Dirección General de Aviación Civil inauguró el Servicio de Vigilancia Radar en el aeropuerto, para lo cual era necesario el cumplimiento de tres requisitos básicos: la implementación tecnológica, la capacitación y el entrenamiento a los controladores de tránsito aéreo, contando hoy por hoy con un nuevo radar que identifica aviones que vuelen muy bajo.

Durante la emergencia sanitaria que vivió nuestro país en torno al coronavirus y la pandemia existente, el Aeropuerto Rio Amazonas debido a su posición estratégica y ayuda social a las comunidades, garantizó la seguridad operacional en los vuelos humanitarios, vuelos militares, vuelos de emergencia médica, vuelos del Estado y vuelos de Aeropolicial, es decir, el aeropuerto nunca dejó de funcionar.

$\mathrm{Su}$ personal técnico y administrativo altamente calificado brindó su contingente permitiendo que las operaciones aéreas se realicen con altos índices de seguridad, garantizando su operación en beneficio de la Amazonia hacia las diferentes pistas autorizadas como: Moretecocha, Curintza, Kapawi, Wiririma, y Montalvo como las principales y Jandiayacu, Llanchamacocha, Lorocachi, Makusar, Masaramo, Nuevo Pindoyacu, Sarayacu, Yusutnza en menor frecuencia, en viajes que duran entre treinta minutos y una hora, pues, influye mucho la distancia de las pistas y la condición climática de la región, fluctuando su precio entre \$900,00USD y \$1440,00USD sin impuestos, ni tasas aeroportuarias.

En lo relacionado a su infraestructura dispone de:

1. Bloque eléctrico terminal aéreo, hangar y oficinas,

2. Oficinas de la UVC RIII,

3. Oficina RSC Amazonas,

4. Oficina electrónica y electricidad,

5. Oficina Ecuafuel y bodega CNS,

6. Aeropolicial, hangar y oficinas SSEI,

7. Bloque eléctrico Torre de control,

8. Torre de control

a. Sala de equipos de comunicaciones aeronáuticas,

b. Meteorología y Torre de control, estructura de 3 pisos 
9. Edificio sala de presentación RADAR

10. Aproximación área social, sala equipos, y SS HH.

11. Parqueadero

Las instalaciones del aeropuerto se encuentran en una zona comercial donde el viajero tiene a su disposición despensas con gran variedad de productos de primera necesidad, alimentos bebidas, refrigerios de todo tipo, además de restaurantes de comida variada, instituciones financieras como Banco General Rumiñahui con un cajero automático que funciona el Brigada de Selva N.17 Pastaza, una sucursal de la CACPE Cooperativa de Ahorro y Crédito de la Pequeña Empresa de Pastaza, y varios cajeros automáticos de otras instituciones financieras que dan el soporte económico al usuario del aeropuerto, es importante mencionar la presencia de farmacias bien equipadas de medicamentos para solventar cualquier necesidad así también, cuenta con una importante planta hotelera para la pernoctación de quienes así lo deseen y transporte terrestre en buses o taxi las 24 horas.

Las cinco compañías de aviación que tienen sus bases de operación en este aeropuerto operan bajo las RDAC Parte 135.- "Requisitos de operación: operaciones domésticas e internacionales regulares y no regulares" y son: Escuelas de Aviación (2), Aviación del Ejército (1), Policía Nacional (1) y Fuerza Aérea Ecuatoriana (1). Es importante señalar que en las instalaciones del aeropuerto se desarrollan las actividades de la Escuela de Aviación Pastaza y la Escuela AEROSERTEC, donde se van formando a los futuros pilotos comerciales.

Los tipos de aeronaves más comunes y de acuerdo a su capacidad que operan en este aeropuerto por las características de las pistas de la Región Amazónica Ecuatoriana son:

Tabla 1: Tipo de aeronave

\begin{tabular}{llr}
\hline N. & TIPO DE AERONAVE & \#PAX \\
\hline 1 & Arava & 23 \\
2 & Cessna 182 & 4 \\
3 & Casa 212 & 26 \\
4 & Cessna 206 & 6 \\
5 & Casa 235 & 43 \\
6 & Piper Azteca & 6 \\
7 & Avión Hércules C130 & 70 \\
8 & Super Puma & 20 \\
9 & Avión Avro & 43 \\
10 & MI-8 & 24 \\
11 & Twin Otter & 19 \\
12 & Gazella & 5 \\
13 & Helicóptero Bell 212 & 9 \\
14 & Kodiak & 9 \\
15 & Cessna 150 & 2 \\
16 & Helio Courier & 6 \\
17 & Cessna 172 & 4 \\
\hline \multicolumn{3}{c}{ Fuente: Informe DAC 2021 }
\end{tabular}

En los últimos cinco años según detalle de operaciones mensuales la DAC jerarquiza (Dirección General de Aviación Civil, 2020) las compañías de la siguiente manera:

Tabla 2: Jerarquización de las compañías

\begin{tabular}{cll}
\hline Nro. & \multicolumn{1}{c}{ Nombre } & \multicolumn{1}{c}{ Observaciones } \\
\hline 1 & SAMAFE (Alas de Socorro del Ecuador) & Transporte de pasajeros y carga \\
2 & AEROKASHURKO & Transporte de pasajeros y carga \\
3 & AEROSARAYACU & Transporte de pasajeros y carga \\
4 & AEROFOR & Transporte de pasajeros y carga \\
5 & AEROCONEXOS & Transporte de pasajeros y carga \\
\hline
\end{tabular}




\begin{tabular}{cll}
\hline & & \\
\hline 6 & TAME & Transporte de pasajeros y carga \\
7 & Escuela AEROSERTEC & Cursos Pilotos de Aviación \\
8 & Escuela de Aviación Pastaza & Cursos Pilotos de Aviación \\
9 & Grupo Aéreo del Ejército 44 Pastaza & Para transporte de personal del Ejército \\
10 & Fuerza Aérea Ecuatoriana & Alas para la integración en la Región Amazónica \\
11 & AEROPOLICIAL & Escuela de Pilotos de helicópteros y atención ECU-911 \\
\hline
\end{tabular}

Fuente: Informe DAC 2020

\section{Análisis estadístico de la encuesta}

Diseño estadístico metodológico: Se procedió a validar sus escalas y variables a través del Alfa de Cronbach posterior al diseño del cuestionario a aplicar. El método de consistencia interna se fundamenta en el Alfa de Cronbach y permite determinar la fiabilidad de un instrumento de medida por medio de un conjunto de ítems que miden el mismo constructo o dimensión teórica (Soler \& Soler, 2012).

Para que el instrumento sea considerado idóneo, y se pueda utilizar con toda la confianza se requiere que cumpla con dos requisitos: confiabilidad y validez" (González, 2008). El valor mínimo aceptable para el coeficiente alfa de Cronbach es 0.7; por debajo de ese valor la consistencia interna de la escala utilizada es baja" (Oviedo \& Campo, 2005). Este valor manifiesta la consistencia interna, es decir, muestra la correlación entre cada una de las preguntas; un valor superior a 0.7 revela una fuerte relación entre las preguntas, un valor inferior revela una débil relación entre ellas. Lucero y Meza (2002) mencionan que "el valor mínimo aceptable del coeficiente de fiabilidad depende de la utilización que se hará del instrumento". Cuanto más cerca se encuentre el valor del Alfa a 1, mayor es la consistencia interna de los ítems analizados.

Los resultados del análisis de fiabilidad para verificar la validez estadística de la encuesta se muestran a continuación:

Fiabilidad, Escala: ALL VARIABLES (según SPSS 22.0)

Tabla 3: Resumen de procesamiento de casos

\begin{tabular}{lrrr}
\hline & & \multicolumn{1}{c}{ N } & \multicolumn{1}{c}{$\%$} \\
\hline Casos & Válido & 385 & 100,0 \\
& Excluido $^{\text {a }}$ & 0 & 0,0 \\
a & Total & 385 & 100,0 \\
La eliminación por lista se basa en todas las \\
variables del procedimiento
\end{tabular}

Tabla 4: Estadísticas de fiabilidad

\begin{tabular}{cc}
\hline Alfa de Cronbach & N de elementos \\
\hline 0,956 & 10
\end{tabular}

El resultado anterior refleja un Alfa de Cronbach con un valor de 0,956 lo cual confirma la fiabilidad y validez del cuestionario ya que dicho valor es muy cercano a 1 . Según criterios de Darren \& Mallery (2003) mencionan que las escalas consideradas en las variables de estudio fueron excelentes además plantean que un coeficiente Alfa de Cronbach por encima o superior a 0.9 se considera excelente. Una vez validada la fiabilidad del instrumento, se analizó la relación entre algunas de las variables 
estadísticamente significativas, por medio de tablas de contingencia, Pruebas de hipótesis Chi-cuadrado y Coeficientes de contingencia. Se consideró el conjunto de variables tomando en cuenta el objetivo de la investigación y los indicadores mencionados para determinar la realidad operativa del aeropuerto:

1. Género

2. Edad

3. Nacionalidad

4. Estado civil

5. Formación académica

6. Profesión

7. De qué aeropuerto viene

8. Ciudad de arribo

9. Lugar de adquisición del pasaje

10. Frecuencia

11. Motivo

12. Número de acompañantes

El análisis de la relación estadísticamente significativa entre las variables antes mencionadas se muestra a continuación, considerando un nivel de significación del 5\% y un nivel de confiabilidad del $95 \%$.

El coeficiente de contingencia es una medida de relación estadística y expresa la intensidad de dicha relación entre dos o más variables nominales u ordinales. Las variables relacionadas entre si, significativamente se usaron para la elaboración de las tablas mostradas que contribuyen a definir la realidad operativa del Aeropuerto Río Amazonas.

Resultados prueba chi cuadrado y coeficiente de contingencia datos "Aeropuerto Río Amazonas"

Tabla 5: Aeropuerto Rio Amazonas Género + Edad + Motivo

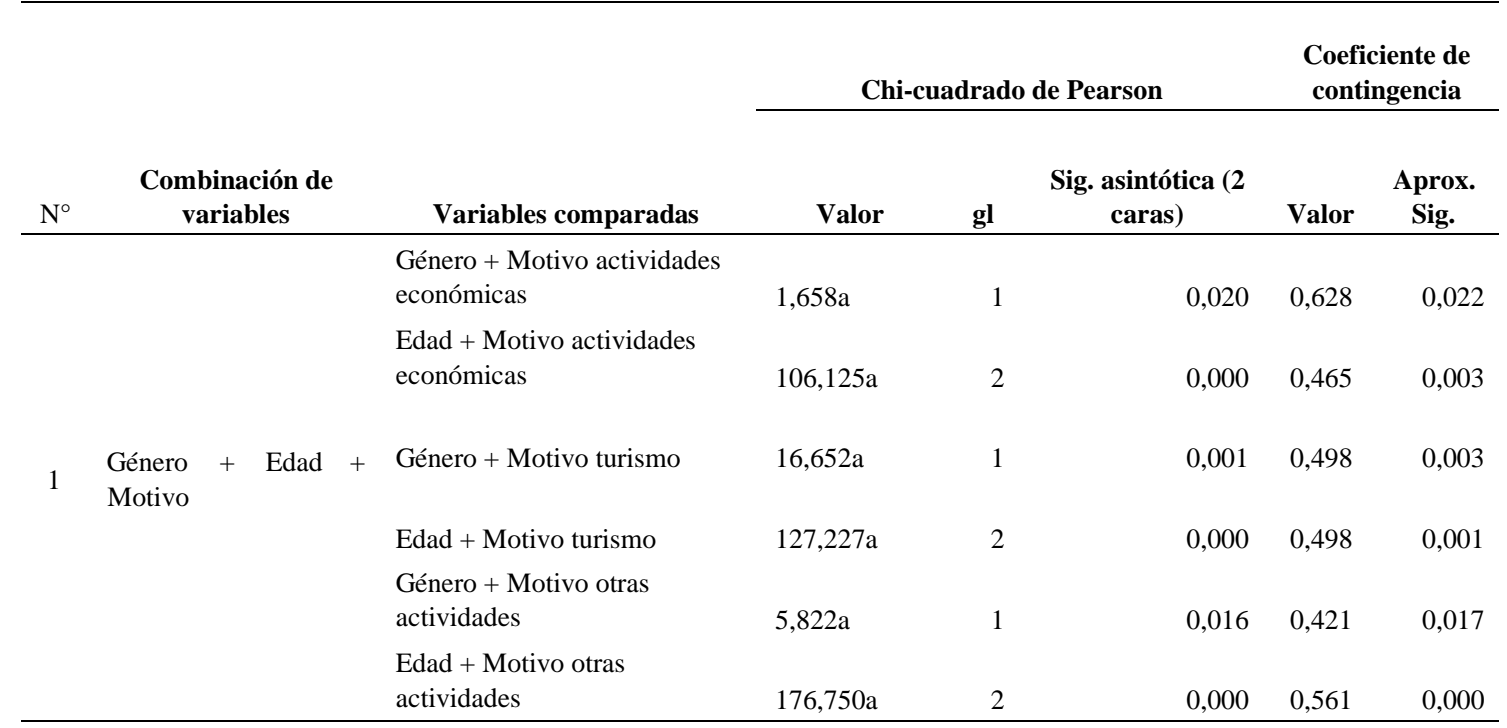


Tabla 5: Aeropuerto Rio Amazonas Género + Edad + Frecuencia

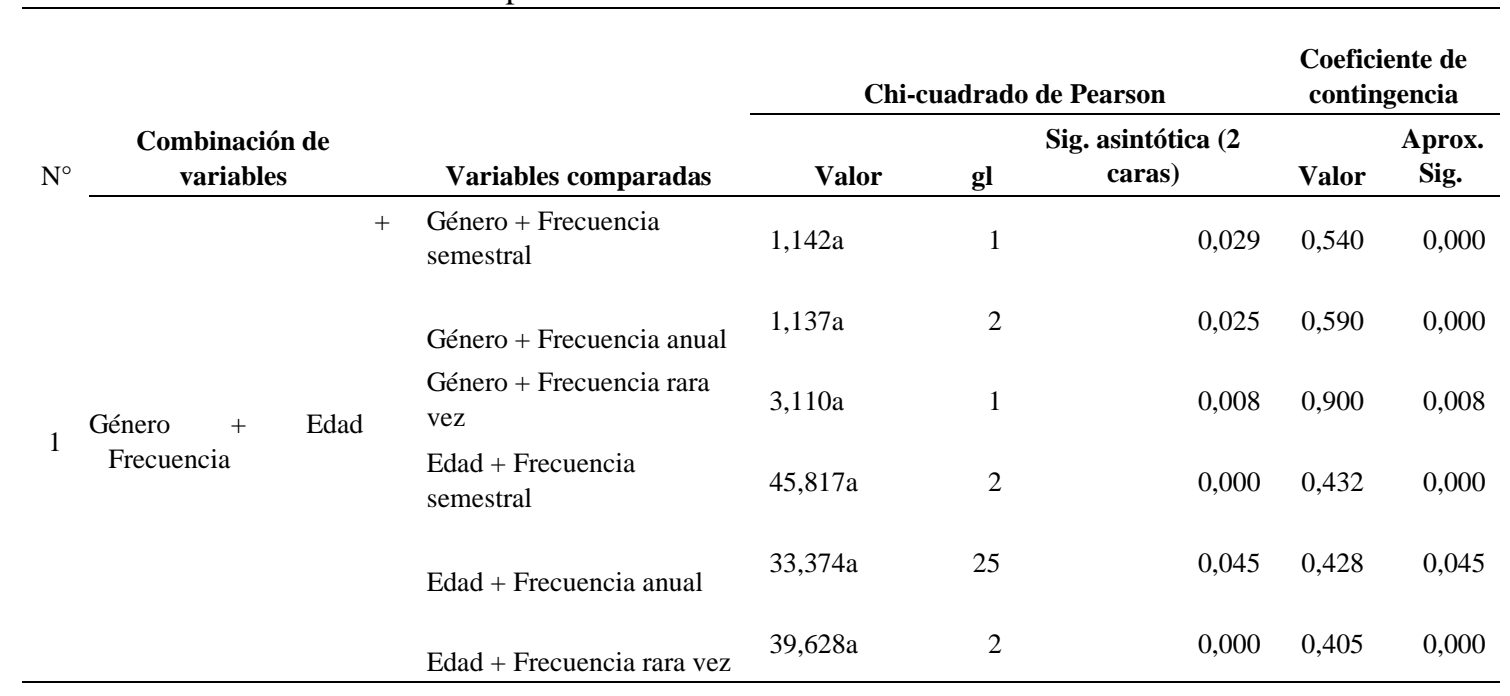

Se muestra que todas las variables están altamente relacionadas entre sí, pues su valor de significación es inferior a 0,05 ; por otro lado, los resultados del coeficiente de contingencia confirman la existencia de relación estadísticamente significativa entre todas las variables analizadas por cuanto sus valores son mayores a 0,40.

\section{Resultados:}

La realidad operativa del tránsito aéreo de pasajeros en este estudio se considera a las bondades del aeropuerto y su efectivo funcionamiento en el tránsito de pasajeros; es de mencionar que las limitaciones de movilidad debido al confinamiento por la pandemia COVID-19 han dificultado el desarrollo del proceso de investigación, sin embargo, se ha cumplido con las expectativas respecto a la participación de los actores de la movilidad del aeropuerto, arrojando los siguientes datos: en cuanto al género se hizo la consulta incorporando masculino, femenino y un tercer ítem de "indeterminado" respetando su identidad de género, sin embargo los resultados muestran a un $57,14 \%$ de mujeres y $42,86 \%$ de hombres; siendo su rango de edad del usuario entre 18 y 35 años, con el 49,4\%, entre 36 y 50 el 31,2 \% y más de 50 el 19.5\%; en lo que respecta al motivo de viaje se ha dividido en 3 importantes grupos que son: por Actividad doméstica que encierra a las actividades propias del hogar, salud, compra de víveres o implementos de casa, turismo que engloba únicamente a pasajeros que usan el aeropuerto por motivo de ocio en ida y vuelta de destinos turísticos exclusivamente, y por otras actividades que son los que ingresan por trabajo como los profesores, gente de las compañías mineras y petroleras en sus diferentes fases de trabajo que ahí laboran, teniendo los siguientes resultados: por actividad doméstica 45,2\%, por otras actividades 49,9.5 y por turismo el 4,9\% confirmando así que el aeropuerto Rio Amazonas depende principalmente de actividades cotidianas del hogar y trabajo en su mayoría, muy poco por turismo; en cuanto a la frecuencia de uso del aeropuerto se consideran las variables de anualmente que obtuvo $24,9 \%$, semestralmente $12,5 \%$ y rara vez el $62,6 \%$; en cuanto a la nacionalidad del usuario son ecuatorianos con un $87,5 \%$, seguido por extranjeros con un $7,3 \%$ y un 5,2 con doble 
nacionalidad; en su estado civil, la mayoría 49,4 son solteros, seguido por casados y divorciados con algo más del $18 \%$ cada indiicador; en su formación académica se evidencia que el 31,2 pertenecen a estudios de tercer nivel, seguido por un $24,2 \%$ de secundaria; seguidamente se evaluó su actividad económica resultando tener la mayoría las personas sin empleo con un $31,2 \%$ y los servidores públicos con un $25,2 \%$, empleados privados con el $18,7 \%$ y estudiantes con el $12,5 \%$; en la pregunta de qué aeropuerto vienen el 68,8\% proceden de la comunidad del interior de la amazonia y el 31,4\% de una ciudad del Ecuador; la adquisición de su pasaje aéreo se realizó en el mismo aeropuerto en un $56,1 \%, 18,7 \%$ otros y 12,5 agencias de viajes; viajeros que mencionan viajar solos en un $31,4 \%, 24,9 \%$ lo hacen con otros acompañantes sea por trabajo, 18,7 en familia y 12,5 con amigos.

Los resultados de esta investigación reflejan el análisis de datos obtenidos sea por fuente primaria y secundaria en el aeropuerto Rio Amazonas con Ayuda de la DAC y los ejecutivos de las aerolíneas que allí operan, en las personas de Ing. José Orbe y al Piloto Rubens Álvarez Benalcázar Jefe de operaciones de AEROKASHURKO por su tiempo y participación en el desarrollo de la investigación.

\section{Conclusiones}

- El Aeropuerto Río Amazonas a noviembre 2020 cuenta con las ventajas competitivas suficientes en cuanto a infraestructura y personal operativo para realizar una operación diaria eficiente hacia el interior de la amazonía y el centro del país.

- Se estableció la realidad operativa del Aeropuerto Río Amazonas a partir de las variables fuertemente relacionadas entre sí, las cuales fueron: género, edad, motivo y frecuencia de uso del aeropuerto, sin embargo, orientan los resultados los demás indicadores.

- Se consolidan los resultados de las bondades del Aeropuerto Río Amazonas para socializar la información actualizada y oportuna al servicio de la comunidad de la amazonia y así mejorar su realidad operativa.

\section{Bibliografía}

Aerosertec. (12 de 12 de 2020). www.aerosertec.ec. Obtenido de www.aerosertec.ec: https://aerosertec.ec/Andina, Comunidad. (18 de 11 de 2019). comunidadandina.org. Obtenido de comunidadandina.org: http://www.comunidadandina.org/DocOficialesFiles/DEstadisticos/SGDE887.p df

Barrantes, R. (2016). Investigación: Un camino al conocimiento un enfoque cualitativo, cuantitativo y mixto. San José de Costa Rica: EUNED.

Boullón, R. (2006). Planificación del espacio turístico. México: Trillas. 
Darren, G., \& Mallery, P. (2003). SPSS for Windows step by step: A Simple guide and reference. 11.0. Boston: Allyn \& Bacon.

Dirección General de Aviación Civil. (2020). Información proporcionada del Sistema SIAO. Quito: DAC.

Dirección General de Aviación Civil. (12 de 12 de 2020). www.ais.aviacióncivil.gob.ec. Obtenido de www.ais.aviacióncivil.gob.ec: http://www.ais.aviacioncivil.gob.ec/designador/SESM

Dirección General de Aviación Civil. (12 de 12 de 2020). www.aviacioncivil.gob.ec. Obtenido de www.aviacioncivil.gob.ec: https://www.aviacioncivil.gob.ec/aeropuerto-rioamazonas-punto-deconectividad-de-320-comunidades-indigenas/

Eyssautier, M. (2006). Metodología de la investigación: Desarrollo de la inteligencia. Mexico: Internacional Thompson.

Fernández, C., Baptista, M., \& Hernández, A. (2006). Análisis de datos. Recuperado el 23 de 4 de 2020, de https://ciberinnova.edu.co: https://ciberinnova.edu.co:10004/archivos/plantilla-ovas1-slide/documentsUCN-Canvas/proyecto-integradorII/lecturas\%20unidad\%202/TEMA\%201/Capitulo\%2010Sampierionlisis\%20de $\% 20$ datos\%20cuantitativos\%20270-335.pdf

González, Y. (2008). Instrumento cuidado de comportamiento profesional: Validez y confiabilidad. AQUICHAN, 170-182.

INEC. (12 de 12 de 2020). www.ecuadorencifras.gob.ec. Obtenido de www.ecuadorencifras.gob.ec: http://www.ecuadorencifras.gob.ec/censo-depoblaciony-vivienda/

Lucero, I., \& Meza, S. (2002). Validación de instrumentos para medir conocimientos. Departamento de Física - Facultad de Ciencias exactas y naturales y agrimensura- UNNE, www1.unne.edu.ar/cyt/2002/09-Educacion/D-027.pdf.

Organización Mundial de Turismo. (06 de 06 de 2012). www2.unwto.org/es. Obtenido de www2.unwto.org/es: http://statistics.unwto.org/es/content/

Oviedo, H., \& Campo, A. (2005). Aproximación al uso del Coeficiente Alpha de Cronbach . Colombiana de Psiquiatría, 572-580.

Rodrigue, J.-P., Comtois, C., \& Slack, B. (2013). The geography of transport. Abingdon: Routledge.

Soler, L., \& Soler, S. (2012). Usos del coeficiente alfa de Cronbach en el análisis de instrumento escritos. Revista médica electrónica, 1-6. 
Veiga, J., De la Fuente, E., \& Zimmermann, M. (2008). Modelos de estudios en investigación aplicada: Conceptos y criterios para el diseño. Medicina y seguridad del trabajo, 81-88.

Visitaecuador. (12 de 12 de 2020). www.visitaecuador.com. Obtenido de www.visitaecuador.com: https://visitaecuador.com/ve/mostrarRegistro.php?informacion=3\&idRegistro=4 68

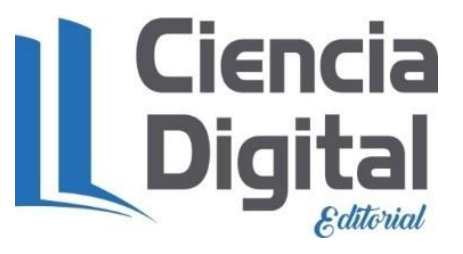




\section{PARA CITAR EL ARTÍCULO INDEXADO.}

González Piedra, B. S., Ruiz Mármol, E. E., Pinta Rodríguez, M. I., \& Espín Ortiz, C. G. (2021). Aeropuerto Río Amazonas y su realidad operativa en el turismo de Pastaza. ConcienciaDigital, $4(2.2)$ 139-152. https://doi.org/10.33262/concienciadigital.v4i2.2.1783

\section{Ciencia LDigital}

El artículo que se publica es de exclusiva responsabilidad de los autores y no necesariamente reflejan el pensamiento de la Revista Conciencia Digital.

El artículo queda en propiedad de la revista y, por tanto, su publicación parcial y/o total en otro medio tiene que ser autorizado por el director de la Revista Conciencia Digital.

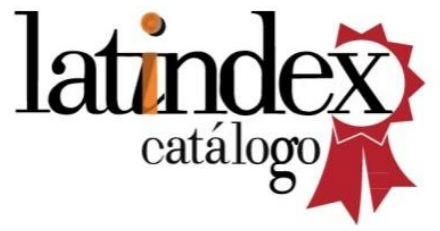

